Relations industrielles

Industrial Relations

\title{
À un carrefour des recours en droit du travail
}

\section{Fernand Morin}

Volume 23, numéro 4, 1968

URI : https://id.erudit.org/iderudit/027957ar

DOI : https://doi.org/10.7202/027957ar

Aller au sommaire du numéro

\section{Éditeur(s)}

Département des relations industrielles de l'Université Laval

\section{ISSN}

0034-379X (imprimé)

1703-8138 (numérique)

Découvrir la revue

Citer cet article

Morin, F. (1968). À un carrefour des recours en droit du travail. Relations industrielles / Industrial Relations, 23(4), 677-683.

https://doi.org/10.7202/027957ar

Tous droits réservés (C Département des relations industrielles de l'Université Laval, 1968
Ce document est protégé par la loi sur le droit d'auteur. L'utilisation des services d'Érudit (y compris la reproduction) est assujettie à sa politique d'utilisation que vous pouvez consulter en ligne.

https://apropos.erudit.org/fr/usagers/politique-dutilisation/ 
mésentente relative à l'interprétation ou à l'application d'une convention collective ». La Cour civile ne peut intervenir pour faire droit à une réclamation de salaire que si aucune difficulté d'interprétation de la convention ne se soulève.

Mais, même ainsi présentée à titre d'exception, cette solution fait naître des craintes relativement à l'intégrité de la compétence de l'arbitre.

Que le tribunal civil admette ainsi les *simples réclamations de salaires », lorsque le salarié en cause est par ailleurs lié par une convention collective, et les exigences de la procédure de réclamation que peut comporter la convention se trouvent dénuées de signification.

Surtout - et c'est ce qui importe - , à qui revient-il vraiment, selon le législateur, de juger si l'on est en présence, ou non, d'une difficulté d'interprétation de la convention? Dans certains cas, la Cour peut bien être d'avis que la contestation de l'employeur est futile; un arbitre aurait pu, à tort ou à raison, apprécier différemment la situation. Or, n'est-il pas celui que le Code du travail charge de l'interprétation de la convention?

Enfin, la définition statutaire du grief ne peut ainsi que se réduire aux seules * mésententes relatives à l'interprétation de la convention collective ». Elle comprend aussi bien les mésententes auxquelles peut donner lieu son "application *. Quelle portée à attribuer à ce dernier terme? Dans le présent arrêt, l'on parle - sans être plus explicite à ce sujet - de clapplicabilité » de la convention. Il faut, en définitive, constater que la convention collective n'est plus tout-à-fait un champ réservé à l'arbitre des griefs.

\section{A un carrefour des recours en droit du travail}

\section{FERNAND MORIN}

En matière de congédiement pour activités syndicales, la C.R.T. est seule compétente. L'arbitre de griefs y retrouve sa compétence lorsque la C.R.T. rejette la plainte. Telle est la règle confirmée récemment par la Cour d'appel de Québec. Pour bien saisir la portée de cette décision, il convient de préciser les données principales de l'affaire et de présenter la position respective de la Cour supérieure et de la Cour dappel. Nous pourrons, par la suite, formuler quelques commentaires.

\section{Donnés PRtncipales de l'affatre.}

Au début d'octobre 1967, un salarié est congédié et formule aussitôt devant la C.R.T. une plainte en congédiement pour activités syndicales 
selon les modalités des art. 14 à 20 C.t. En février 1968, la C.R.T. convoque les parties à une séance d'audition prévue pour le 2 avril 1968 (six mois après le congédiement). Entre-temps, soit le 2 novembre 1967, le syndicat présentait un grief pour cette même affaire, en suivant la procédure établie à la convention collective. Signalons que cette convention collective prévoyait expressément qu'une telle mésentente pouvait être objet de grief :

6.02 Dans aucun cas, il ne sera permis à un employeur de discipliner, suspendre, congédier ou refuser d'embaucher un travailleur pour activités syndicales ou pour son statut de membre du Syndicat. s

- 10.01 Tout employé ancien ou nouveau, qui se croit lésé dans son droit ou injustement traité, peut formuler une plainte ou présenter un grief pour enquête et règlement dans les huit $(8)$ jours ouvrables qui suivent la naissance ou la connaissance de la plainte ou grief; toute plainte ou grief devra être soumis de la façon suivante :

a) tout employé ayant un grief, seul ou accompagné de son aģent d'affaires, ou son agent de griefs ou toute personne autorisée par le Syndicat soumettra verbalement le grief au contremaître ou à l'employeur;

b) si le contremaitre ou l'employeur, selon le cas, ne peut régler le grief de façon satisfaisante dans les deux (2) jours ouvrables suivants, l'agent d'affaires pourra recourir à l'arbitrage en la manière prévue à l'art. 88 du Code du travail (12-13 Elizabeth II, chapitre 45).

Conformément aux modalités de la clause 10.01, le ministre du travail nomma un arbitre pour ce grief. La première séance de l'arbitrage était prévue pour le 4 juin 1968. Pour laisser place à la procédure arbitrale, le syndicat demanda à la C.R.T. la remise de l'audition fixée pour le 2 avril.

\section{Déctsions de la Cour supérieure et de la Cour d'APPei.}

\section{- d la Cour supérieure :}

En raison de ce double recours, l'employeur présenta à la Cour supérieure une requête pour l'émission d'un bref enjoignant l'arbitre d̀ se dessaisir du grief :

- La requérante allègue que la Commission des relations du travail de Québec a juridiction exclusive pour entendre ce litige, que cette affaire ne peut être entendue par deux organismes quasi judiciaires différents, avec possibilité de décision contradictoires. 1

(1) Ludger Harvey \& Fils Ltée v. Monsieur larbitre Laurent Cossette, avocat et Le Syndicat de lindustrie de la Construction de Jonquière Inc. et M. Almas Tremblay - Cour supérieure, district de Chicoutimi, no 40-024, pages 2 et 3 , décision de M. le juge en chef Frédéric Dorion. 
Au point de départ, le juge en chef reconnaît la compétence exclusive de la C.R.T. pour entendre et décider de toute plainte où on prétend à un congédiement pour activités syndicales :

- C'est l'article 14 du Code du travail qui donne à la Commission des relations du travail, juridiction pour entendre une plainte portée par un salarié qui a été congédié «à cause de l'exercice par ce salarié d'un droit qui lui résulte du présent Code.

- D'où il découle que si un salarié est congédié pour activités syndicales, c'est bien la Commission des relations du travail qui a juridiction exclusive pour entendre le litige et qui, après avoir fait l'enquête sur les faits, décidera si réellement les actes posés par le salarié sont de nature à justifier son congédiement. ${ }^{2}$

Monsieur le juge Frédéric Dorion analyse la portée de l'art. 88 du Code du travail en regard de la définition du grief, selon l'art. 1-(g) C.t. :

- Il est bien évident que le congédiement d'un salarié n'est pas, à proprement parler, une mésentente relative à l'interprétation d'une convention. D'autre part, le droit qu'a un salarié de faire des activités syndicales est prévu aux articles 3 et suivants, et il semble bien que c'est lorsque ce droit est discuté qu'il faut alors appliquer les art. 14, 15 et 16. Il est vrai que l'art. 6.02 de la convention se rapporte à un congédiement possible d'un salarié. Mais, la convention ne peut affecter les dispositions de la loi.»

En conséquence, le juge en chef de la Cour supérieure conclut ainsi :

«... si l'on en vient à la conclusion qu'un litige entre un salarié et un employeur proviendrait des activités syndicales du salarié, c'est l'art. 14 du Code qu'il faut appliquer, même si dans la convention il est question, comme nous venons de le voir, de congédiement. Il ne peut y avoir de doute que la loi prime la convention et que ce n'est qu'à défaut d'une disposition de la loi que l'on peut avoir recours a la convention. $>3$

Si la C.R.T. décide qu'il ne s'agit pas d'un congédiement pour sctivités syndicales, alors :

-... dans ce cas, il y aura toujours lieu pour la Commission de décliner sa juridiction, pour qu'ensuite les griefs soient examinés par un arbitre. $\$$

(2) Jugement de la Cour supérieure, déjà cité, p. 4.

(3) Cour supérieure, op. cit, p. 4.

(4) Cour supérieure, op. cit., p. 5. 


\section{- a la Cour dappel :}

En vertu de l'art. 122 du Code du travail, la décision de la Cour supérieure fut portée en appel. La Cour d'appel, formée alors de deux juges, confirma la décision de la Cour supérieure :

- Par sa requête devant la Commission, Tremblay invoquait un droit reconnu par le Code, et il appartenait à la Commission de décider si l'employeur avait repoussé la présomption créée en faveur de l'employé et qu'en fait ce dernier avait été congédié pour d'autres causes que celle de ses activités syndicales. L'arbitre appelé à juger de la justice du congédiement devra nécessairement décider dans le cas où les prétentions de l'employeur sont maintenues, qu'en fait Tremblay n'avait pas été congédié pour activités syndicales, que les autres motifs de congédiement seraient prouvés et le justifieraient. ${ }^{\circ}$

En somme, la Cour d'appel affirme la nécessité de donner priorité à la C.R.T. parce qu'elle a compétence exclusive en vertu d'une disposition législatice (art. 14 à 20 C.t.) pour décider sur le motif syndical invoqué par le salarié :

- A ce stade des procédures, le recours devant l'arbitre serait le même que celui présenté devant la Commission, et comme il semble à l'examen des motifs de congédiement, tant dans la plainte du 6 octobre que dans le grief invoqué par le Syndicat, qu'il s'agit d'abord de décider si Tremblay a été congédié pour activités syndicales ou pour d'autres motifs, la Commission des relations de travail du Québec a juridiction exclusive pour décider de cette question. . (p. 7).

Puisque l'arbitre n'a pas compétence en cette matière, l'article 121 C.t. (exclusion au contrôle judiciaire) n'empêche pas alors les tribunaux de rappeler les limites de sa juridiction. Sur ce point, la Cour d'appel rappelle deux décisions : l'arrêt Komo Construction Inc. et al vs. Commission des relations de travail du Québec et al, 1968 R.C.S. 172 et Alliance des professeurs catholiques de Montréal vs. Labour Relations Board, 1953 R.C.S. 140.

\section{Commentaire}

Selon cette décision, la C.R.T. doit d'abord établir et, d'une façon exclusive, si le congédiement résulte des activités syndicales du salarié. Conformément aux dispositions édictées aux art. 14 à 20 C.t., une

(5) Laurent Cossette, arbitre, requérant v. Ludger Harvey \& Fils Limitée, intimé et le Syndicat de l'Industrie de la Construction de Jonquière Inc., et Almas Tremblay, Cour d'appel, district de Québec, no 7700, 11 sept. 1968, pp. 6-7. 
réponse affirmative de la C.R.T. peut signifier l'émission d'une ordonnacne en réintégration et le paiement d'une indemnité équivalente à la perte réelle du salaire. En ce cas, il ne peut être question, par la suite, d'exercer un deuxième recours. Mais, le rejet de la plainte par la C.R.T. ne signifie pas que le congédiement fut pour une cause juste at suffisante; cette décision établira seulement que le congédiement ne fut pas décidé en raison des activités syndicales du salarié. Ainsi, à la suite de cette réponse négative de la C.R.T., les tribunaux ont reconnu que l'arbitre de griefs pouvait être valablement saisi de l'affaire. A ce moment, l'arbitre doit décider si le congédiement est pour une cause juste et suffisante (art. 13, in fine C.t.). Le renvoi à l'arbitre de griefs doit se faire selon les dispositions de l'art. 88 C.t. et les modalités établies à la convention collective. Selon nous, cette approche respecte bien l'économie du droit du travail. Les articles 14 à 20 C.t. pourvoient un mécanisme simple et réaliste (le fardeau est facilement mis à la charge de l'employeur) et les moyens de réparation (réintégration et indemnité pour perte de salaire) dont est dotée la C.R.T. sont suffisamment drastiques et efficaces. Pour ces seules raisons et en plus des considérations strictement juridiques, il nous paraît déjà préférable que toute plainte portant sur une quelconque atteinte à la liberté syndicale relève exclusivement de la C.R.T. Ni l'employeur, ni le syndicat, ni même le salarié en cause ne peuvent maintenant discuter sur ce point : seule la C.R.T. a compétence exclusive pour entendre et décider d'une telle plainte. Malgré la clarté de cette règle, la décision de la Cour supérieure nous incite à soulever trois autres questions.

- La prescription du grief : Au terme du premier recours, devant la C.R.T., le droit du salarié à formuler pareil grief peut-il être prescrit? Le cas à l'étude illustre bien l'importance de la question. Rappelons que l'audition devant la C.R.T. eut lieu six (6) mois après le congédiement. Or, l'art. 59 C.t. édicte la règle suivante :

\footnotetext{
- Les droits et recours qui naissent d'une convention collective ou d'une sentence qui en tient lieu se prescrivent par six (6) mois à compter du jour où la cause de l'action a pris naissance. Le recours à la procédure de griefs interrompt la prescription. .
}

De plus, le para. 10.01 de la convention collective obligeait le salarié à présenter son grief dans les huit (8) jours ouvrables qui suivent le congédiement. A n'en point douter, ce problème de la double procédure (à la C.R.T. puis, devant l'arbitre des griefs) n'est pas expressément prévu au Code. Certes, la convention collective pourrait prévoir cette situation à la procédure de griefs. Aussi, le Lieutenant-gouverneur en Conseil, sous lempire de l'art. 91 C.t., pourrait édicter quelques dispositions précises de façon à éviter cette difficulté. Entre temps, nous croyons qu'il serait prudent pour le syndicat ou le salarié, selon le cas, de formuler en temps utile, le grief tout en ayant présenté une plainte 
devant la C.R.T. selon les prescriptions de l'art. 15 C.t. Avant la tenue de l'audition du grief ou au début de la première séance, il est possible de demander à l'arbitre de griefs de surseoir jusquà ce que la C.R.T. ait rendu sa décision. De cette façon, nous croyons que la prescription de six mois serait interrompue. Dans une sentence arbitrale, monsieur le juge Jean-Charles Simard reconnaissait que la formulation écrite d'une plainte était suffisante pour interrompre la prescription, comme le prévoit l'art. 59. C.t. ( $*$ le recours à la procédure de griefs interrompt la prescription s) :

- Même si nous faisons abstraction des circonstances de temps qui ont donné ouverture au grief actuel, il nous faut encore admettre d'une part que cette formulation écrite d'une plainte à la requérante demeurait clairement indicative de l'intention de monsieur Ouellet, de soulever un grief en rapport avec son congédiement..., ${ }^{\circ}$

Cette approche ne répugne pas, croyons-nous, puisqu'il s'agit de deux procédures différentes et complémentaires et dont l'une est prioritaire : déterminer par la C.R.T. s'il s'agit d'un congédiement pour activités syndicales et, si non, déterminer par l'arbitre si c'est pour une autre cause juste et suffisante.

- L'arbitralité du congédiement: Monsieur le juge Frédéric Dorion exprime un autre point que nous ne pouvons négliger:

- Il est bien évident que le congédiement d'un salarié n’est pas, à proprement parler, une mésentente relative à l'interprétation d'une convention. >

Cet orbiter dictum, selon nous, indique bien que l'arbitre peut être saisi d'un grief en congédiernent dans la mesure seulement où on peut $\mathrm{y}$ trouver un fondement à la convention collective. C'est-à-dire que l'arbitre aurait juridiction pour entendre pareille affaire s'il s'agit bien d'un grief au sens du Code du travail [une mésentente relative à l'interprétation ou à l'application de la convention collective $(1-(\mathrm{g})]$ ou encore, s'il s'agit d'un grief selon une définition élargie donnée à la convention, en vertu de l'art. 90 C.t. Le moins que l'on puisse dire, c'est que toute mise à pied, tout congédiement, toute décision de l'employeur à l'égard des salariés n'est pas automatiquement et nécessairement de la compétence de l'arbitre de griefs. Ce dernier devrait donc toujours être prudent et s'assurer de l'arbitrabilité du grief qu'on entend lui soumettre. Il peut, selon nous, soulever de son propre chef, cette question.

(6) Syndicat des employés municipaux de la Ville de Chicoutimi c. la Ville de Chicoutimi, 1966 R.D.T. 65, à la page 75, ler sept. 1965. 
- Pour une justice expéditive: Cette affaire met également en lumière une autre lacune de notre système. Nous constatons que la C.R.T. fixa la première séance d'audition six mois après la réception de la plainte (congédiement le 5 oct. 1967 et la date prévue pour l'audition fut le 2 avril 1968). Pour le salarié qui croit qu'il sera réintégré, pour l'employeur susceptible d'être contraint de reprendre ce salarié et enfin, en raison de la difficulté de coordonner les deux recours, et des conséquences qui peuvent en résulter, il est essentiel que la C.R.T. entende et décide des cas avec beaucoup plus de célérité. N'est-ce pas d'ailleurs pour cette raison que le Syndicat de l'industrie de la Construction de Jonquière Inc., décida, au mois de novembre, soit un mois après le congédiement, de présenter le grief à l'arbitrage. Si la C.R.T. a compétence exclusive sur un point et, si elle doit avoir préséance sur l'arbitre de griefs, ne convient-il pas qu'elle procède avec diligence pour éviter que pendant près d'un an, des salariés soient à tord ou à raison, sur la * corde à linge *? Quelle que soit la rigueur de la décision de la C.R.T. en pareil cas, l'employeur et le salarié doivent savoir, le plus rapidement possible, quels sont leurs droits et leurs obligations respectifs. L'élément temps est toujours, et, d'une façon plus spéciale en droit du travail, très important à l'administration d'une justice véritable. Nous rroyons que toute affaire basée sur l'art. 14 C.t. devrait être décidée clans un délai non supérieur à trois (3) mois.

\section{LE CODE DU TRAVAIL DU QUÉBEC (1965)}

Lo genèse du présent Code du travail (GERARD HEBERT). Le Droit d'ossociation, son extension, ses limites (J.-R. CARDIN). La Commission des Relations de Travail (ANDRE ROY). La Commission des Relations de Travail et l'accréditation syndicale (GERARD VAILLANCOURT). Le Code du travoil et la convention collective (FERNAND MORIN), a procédure de négociation et le recours à la grève ou ou lockout (MARIUS BERGERON). Le règlement pacifique des conflits (ROBERT AUCLAIR). Points de vue sur le Nouveau Code du trovail (ROGER THIBAUDEAU), (LOUIS LABERGE), (DENIS GERMAIN), (J.-P. DESPRES), (ANDRE DEOM), (LAVAL GRONDINES), (RAYMOND PARENT), (LS-CLAUDE TRUDEL). Le nOUveau rôle du Ministère du travail (HON. CARRIER FORTIN). Code du Trovoil. Bill 55 - Loi de la fonction publique.

1 volume, 264 pages - Prix: $\$ 4.00$

LES PRESSES DE L'UNIVERSITÉ LAVAL

Cose Postole 2447

Québec 2

Tél.: 656-2131 